\title{
Optimization of Enzyme Digestion Conditions for Quantification of Glycated Hemoglobin Using Isotope Dilution Liquid Chromatography-Tandem Mass Spectrometry
}

\author{
Ji-Seon Jeong* \\ Center for Bioanalysis, Department of Metrology for Quality of Life, Korea Research Institute of Standards and Science, \\ Daejeon 305-340, Korea
}

Received April 21, 2014; Revised June 05, 2014; Accepted June 05, 2014

First published on the web June 30, 2014; DOI: 10.5478/MSL.2014.5.2.52

\begin{abstract}
Glycated hemoglobin (HbA1c) is used as an index of mean glycemia over prolonged periods. This study describes an optimization of enzyme digestion conditions for quantification of non-glycated hemoglobin (HbA0) and HbAlc as diagnostic markers of diabetes mellitus. Both $\mathrm{HbA} 0$ and $\mathrm{HbAlc}$ were quantitatively determined followed by enzyme digestion using isotope dilution liquid chromatography-tandem mass spectrometry (ID-LC-MS/MS) with synthesized $N$-terminal hexapeptides as standards and synthesized isotope labeled hexapeptides as internal standards. Prior to quantification, each peptide was additionally quantified by amino acid composition analysis using ID-LC-MS/MS via acid hydrolysis. Each parameter was considered strictly as a means to improve digestion efficiency and repeatability. Digestion of hemoglobin was optimized when using $100 \mathrm{mM}$ ammonium acetate (pH 4.2) and a Glu-C-to-HbA1c ratio of $1: 50$ at $37^{\circ} \mathrm{C}$ for $20 \mathrm{~h}$. Quantification was satisfactorily reproducible with a $2.6 \%$ relative standard deviation. These conditions were recommended for a primary reference method of $\mathrm{HbA1c}$ quantification and for the certification of $\mathrm{HbA} 1 \mathrm{c}$ reference material.
\end{abstract}

Keywords: glycated hemoglobin, isotope dilution LC-MS/MS, enzyme digestion, diabetes mellitus

\section{Introduction}

Glycated hemoglobin is a form of hemoglobin used as a control index for diabetes mellitus, and measures the average plasma glucose concentration over a prolonged period. Hemoglobin $\mathrm{A}_{1 \mathrm{c}}(\mathrm{HbA1c})$ is the major component of glycated hemoglobin, which is formed by the nonenzymatic attachment of glucose to the $N$-terminal valine of the $\beta$-chain of hemoglobin. The life span of erythrocytes is $\sim 120$ days, allowing HbA1c to reflect average glucose exposure over the preceding 8-12 weeks. ${ }^{1-3}$

There are more than 30 assay methods to measure $\mathrm{HbA1c}$, ${ }^{4,5}$ causing interlaboratory variability, and thus, an effort to standardize. To achieve uniform international standardization, the international federation of clinical

\section{Open Access}

*Reprint requests to Ji-Seon Jeong

E-mail: jsjeong@kriss.re.kr

All MS Letters content is Open Access, meaning it is accessible online to everyone, without fee and authors' permission. All MS Letters content is published and distributed under the terms of the Creative Commons Attribution License (http://creativecommons.org /licenses/by/3.0/). Under this license, authors reserve the copyright for their content; however, they permit anyone to unrestrictedly use, distribute, and reproduce the content in any medium as far as the original authors and source are cited. For any reuse, redistribution, or reproduction of a work, users must clarify the license terms under which the work was produced. chemistry and laboratory medicine (IFCC) has established a working group to coordinate activities worldwide. ${ }^{6-8}$ The working group has developed a reference system, a reference measurement method, ${ }^{9}$ as well as reference material ${ }^{10}$ to be used as the basis for international standardization. It quantified $N$-terminal hexapeptides of the $\beta$-chain of human hemoglobin for HbA0 (VHLTPE; Hexa) and of 1deoxyfructosyl-VHLTPE (G-Hexa) for HbAlc. The difference in mass by the glucose at the $N$-terminal of $\mathrm{HbA1c}$ allows separation and quantification by HPLC and MS, respectively. Based on the IFCC reference measurement procedure, Kaiser et $\mathrm{al}^{11}$ developed an isotope dilution-mass spectrometry (ID-MS) method by using synthetic isotope labeled $N$-terminal hexapeptides of the $\beta$-chain as internal standards. Because ID-MS is a method of the highest metrological order, the ID-LCMS procedure for $\mathrm{HbAlc}$ analysis allows the reference measurement procedure to be raised to a higher order of accuracy. Even if the ID-MS method could guarantee accurate quantification, enzyme digestion should be controlled to minimize diagnostic errors in the clinic. Because the enzyme reaction is sensitive to experimental conditions, the quality of enzyme digestion is the critical regulation step in this procedure. ${ }^{12,13}$

In this study, some parameters of enzyme digestion for HbA1c were optimized. Those were the quantity and volume of sample and enzyme, $\mathrm{pH}$ of buffer, dilution reagent, and enzyme variation. Additionally, the synthetic peptides were 
certified using amino acid (AA) analysis via acid hydrolysis to establish traceability to SI units and to ensure the accuracy of HbAlc quantification. Optimized sample preparation procedures were applied to produce $\mathrm{HbAlc}$ certified reference material (CRM). This method could be applied in the development of similar proteolytic digestion procedures.

\section{Experimental}

\section{Preparation of hemoglobin sample}

Human blood hemolysate with an indicative value of $62.9 \pm 1.8 \mathrm{~g} / \mathrm{kg}$ (high resolution HPLC; MonoSTM) was purchased from IRMM (Geel, Belgium) ${ }^{14}$ and reconstituted with distilled water following the manufacturer's instructions. It was then divided into $100 \mu \mathrm{L}$ aliquots for single use, and stored at $-70^{\circ} \mathrm{C}$ before use. Total hemoglobin concentration was $15 \mathrm{~g} / \mathrm{L}$ after reconstitution as indicated by the manufacturer.

\section{Chemicals and reagents}

Ammonium acetate (AmAc), formic acid (FA), and endoproteinase Glu-C were purchased from Sigma-Aldrich (St.Louis, MO, USA). Hemolyzing reagent (Tina-quant HbAlc Gen. 2) was purchased from Roche Diagnostics (Rotkreuz, Switzerland). The AA mixture used for calibration was a CRM comprising AAs in $0.1 \mathrm{M}$ hydrochloric acid (HCl) from the NIST (2389a; Gaithersburg, MD, USA). Isotope labeled AAs L-proline (U${ }^{13} \mathrm{C}_{5}, 98 \% ;{ }^{15} \mathrm{~N}, 98 \%$; Pro*) and L-leucine (U- ${ }^{*} \mathrm{C}_{6}, 98 \%$; ${ }^{15} \mathrm{~N}, 98 \%$;Leu*) were obtained from Cambridge Isotopes Laboratory (Andover, MA, USA). Hydrochloric acid ( $\mathrm{HCl})$ was purchased from Junsei (Tokyo, Japan), and acetonitrile (ACN) was purchased from Thermo Fisher Scientific (Waltham, MA, USA). Other reagents and solvents used were of analytical grade. Water used to prepare the standard solutions, sample solutions, and mobile phase was purified using a Millipore Alpha-Q water purification system (Millipore, Billerica, MA, USA), and solvents were filtered through a membrane filter (pore size $0.2 \mu \mathrm{m}$ ).

The synthetic peptides Hexa (694.36 Da), VH[ $\left.d_{7}\right] \mathrm{LTPE}$ (Hexa*; 701.36 Da), G-Hexa (856.50 Da), and GVH $\left[d_{7}\right]$ LTPE (G-Hexa*; $\left.863.50 \mathrm{Da}\right)$ were synthesized by Peptron (Daejeon, Korea).

\section{Peptide hydrolysis and amino acid analysis}

Two hundred microliters of VHLTPE (Hexa) and GVHLTPE (G-Hexa) solution as well as $200 \mu \mathrm{L}$ of isotopelabeled AA mixture were placed gravimetrically into a glass tube $(15 \mathrm{~mL}$, PYREX screw cap culture tubes with Teflon-lined phenolic caps, cat \# 9826-16x; Corning, NY, USA) and mixed well. A final concentration of $8 \mathrm{M}$ was reached by addition of $1.6 \mathrm{~mL}$ of $10 \mathrm{M} \mathrm{HCl}$. The tubes were sealed, the solutions mixed, and peptides hydrolyzed at $130^{\circ} \mathrm{C}$ for $6 \mathrm{~h}$. The acid was evaporated under nitrogen gas and the residue was dissolved in $400 \mu \mathrm{L}$ of $0.1 \mathrm{M} \mathrm{HCl}$, filtered through a disposable syringe filter, and then injected into the LC-MS system. The AA analysis by liquid chromatography-tandem mass spectrometry (ID-LC-MS/ MS) was carried out as per Jeong et al. ${ }^{15}$ The certified hexapeptide solutions were divided into small aliquots of $100 \mu \mathrm{L}$ for single use, and stored at $-70^{\circ} \mathrm{C}$ before use.

\section{LC-MS conditions for peptide analysis}

The LC-MS/MS analysis was performed using an ACQUITY series UPLC system coupled in-line to a Xevo TQ-S MS system (Waters, Massachusetts, USA). Chromatographic separation was performed using a KINETEX $\mathrm{C}_{18}$ column $(150 \mathrm{~mm} \times 2.1 \mathrm{~mm} \mathrm{I.D.,} 2.6 \mu \mathrm{m}$ particle size; Phenomenex, Torrance, CA, USA) connected to a guard column (Security Ultra, Phenomenex). Mobile phase A comprised $0.1 \%(\mathrm{v} / \mathrm{v})$ FA and mobile phase B comprised $0.1 \%(\mathrm{v} / \mathrm{v}) \mathrm{FA}$ in ACN. Gradient elution was performed using mobile phases $\mathrm{A}$ and $\mathrm{B}$ at a flow rate of $300 \mu \mathrm{L} / \mathrm{min}$ as follows: The initial condition was $94 \%$ mobile phase A. After injection, a linear gradient to $89.8 \%$ mobile phase A was reached over 5 min. The column was cleaned with $30 \%$ mobile phase A for $1.5 \mathrm{~min}$, then returned to initial conditions and re-equilibrated for $1 \mathrm{~min}$. Total run time was $7.5 \mathrm{~min}$ per $3 \mu \mathrm{L}$ injection, and the column oven temperature was maintained at $40^{\circ} \mathrm{C}$. The peptides were quantified on a triple-quadrupole mass spectrometer equipped with a turbospray in multiple reaction monitoring (MRM) mode. The precursor-toproduct ion transitions $m / z 695.4>110.1$ and 237.2 (Hexa); $m / z 702.4>110.1$ and 237.2 (Hexa*); $m / z$ 857.5 $>110.1$ and 220.2 (G-Hexa); and $m / z 864.5>110.1$ and 220.2 (GHexa*). Source ionization and fragmentation parameters were finely tuned by monitoring the MS signal prior to sample analysis. Peaks of unlabeled and isotope-labeled compounds were integrated and their ratio was calculated. The peak area ratios were calculated against the peak area ratio of the standard, which had molar peptide masses close $(<10 \%$ difference) to that of the sample solution.

\section{Sample preparation}

The reconstituted sample was taken from the refrigerator and equilibrated at room temperature for at least $1 \mathrm{~h}$, then diluted tenfold with distilled water. Ten microliters of working sample was placed into a vial and mixed with $30 \mu \mathrm{L}$ of $100 \mathrm{mM}$ AmAc buffer (pH 4.2) fortified with isotope-labeled peptide standards. The solution was well mixed and centrifuged for spin-down. Ten microliters of $0.2 \mathrm{mg} / \mathrm{mL}$ Glu-C was added to the sample and the reaction was kept at $37^{\circ} \mathrm{C}$ for $20 \mathrm{~h}$ under gentle agitation. The final sample volume was $50 \mu \mathrm{L}$, and the mass ratio of Glu-C-toHbA1c was approximately 1:50 in this study.

Each parameter of enzyme digestion such as buffer $\mathrm{pH}$, enzyme-to-sample ratio, dilution reagent, and enzyme type was optimized for accuracy and precision of measured values and for reproducibility. 


\section{Results and discussion}

\section{Determination of standard peptides}

The synthetic peptides were determined by AA analysis coupled with ID-LC-MS/MS via acid hydrolysis. Prior to determination, the hydrolysis conditions were optimized for the object peptides. Through the optimization process, hexapeptides were found to be optimally hydrolyzed when treated with $8 \mathrm{M} \mathrm{HCl}$ at $130^{\circ} \mathrm{C}$ for $6 \mathrm{~h}$ at a $5 \mu \mathrm{mol} / \mathrm{kg}$ sample concentration. Because the chemical properties of each residue are different, the hydrolysis rates and stabilities can be altered under various hydrolysis conditions. ${ }^{16}$ Consequently, the results agreement between different residues can represent the reliability of the hydrolysis process. In this study, the AAs Pro and Leu were determined from hexapeptides hydrolysate. The ratio of unlabeled AA to isotope labeled AA versus the hydrolysis time reached a plateau when the hydrolysis was continued for more than $6 \mathrm{~h}$. This was similar with G-Hexa (data not shown). In a previous study, human growth hormone was completely hydrolysed over $48 \mathrm{~h}$ under the same conditions, ${ }^{15}$ leading us to believe that molecular size influences hydrolysis time.

\section{Separation of Hexa and G-Hexa}

Hexa and G-Hexa were clearly separated by gradient elution within $8 \mathrm{~min}$. Figure 1 shows typical total ion chromatogram (TIC) (A) and MRM scans (B) of the standard mixture of Hexa and G-Hexa. Total run time, including re-equilibration, was $7.5 \mathrm{~min}$, and that was 3 times shorter than that used in the IFCC reference measurement procedure. ${ }^{9}$ Each injection has cleaning step in the gradient elution program using an increased solvent strength to elute highly nonpolar compounds, such as additional proteolytic peptides and proteins from enzymes or hemolysates. In some applications of UPLC using a fast flow rate, the lack of sufficient time for mass transfer can cause carryover and resolution degradation. A blank sample was injected after each sample injection to check for carryover, and no additional peaks were detected in the eluent of the blank.

\section{Optimization of the enzymatic digestion}

The HbA1c samples were digested in AmAc buffer solution (pH 4) at $37^{\circ} \mathrm{C}$ overnight (longer than $18 \mathrm{~h}$ ). Based on these conditions, the specific points that could affect repeatability and reliability in enzyme digestion were investigated. Glu-C is a serine endoproteinase, that hydrolyzes peptide bonds on the carboxyl side of glutamyl and aspartyl residues. ${ }^{17}$ The enzyme preferentially cleaves glutamyl bonds in both AmAc buffer and ammonium bicarbonate buffer ( $\mathrm{pH} 8)$. Both were tested for the greatest signal intensity and stable results. The AmAc buffer was selected as the superior choice (data not shown). Using this buffer, the enzyme-to-HbAlc ratios were tested across a wide range, from $1: 10$ to $1: 100$, in different sample volumes. Figures $2 \mathrm{~A}$ and $\mathrm{B}$ summarize the results. While the signal intensities of Hexa and G-Hexa, as well as the GHexa-to-Hexa ratio fluctuated when the sample volume was small $(<50 \mu \mathrm{L})$, the results of higher volume samples was repeatable. The results of enzyme-to-HbA1c ratios greater than 1:50 were not significantly increased. This means that the enzyme-to-HbA1c ratio was not critical to quantification because Glu-C had a wide range of enzyme activity, covering a wide range of proteins. To reduce the consumption of costly Glu-C, a 1:50 (approximate) ratio of Glu-C-to-HbA1c was recommended.

In some cases, an analytical bias can occur in standards

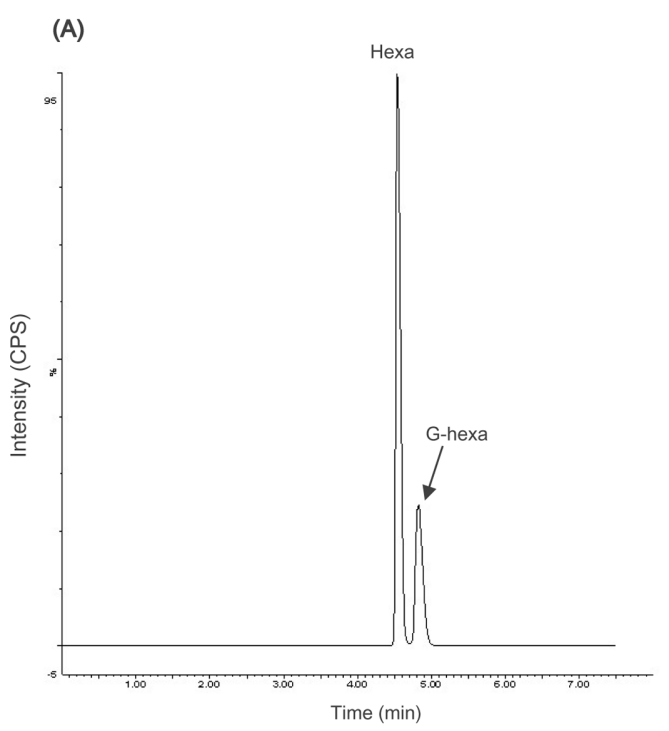

(B)

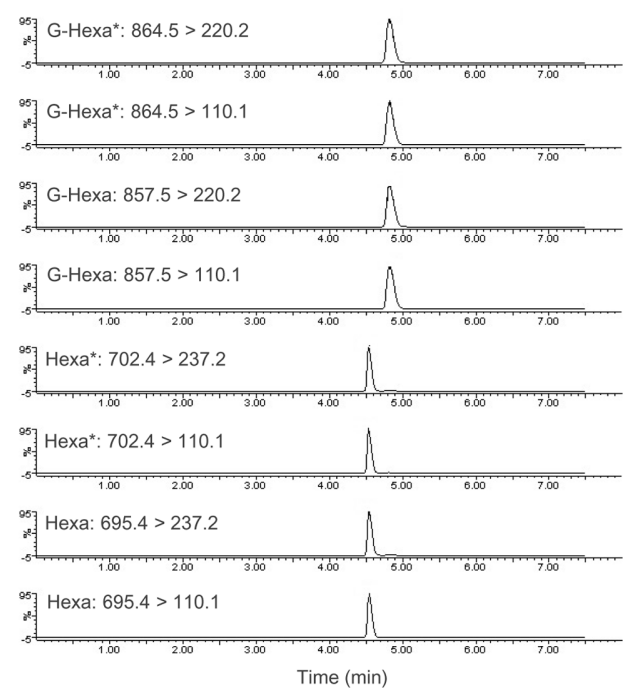

Figure 1. Typical TIC (A) and MRM (B) scans of $5 \mu \mathrm{M}$ Hexa and G-Hexa standard mixtures. 
(A)

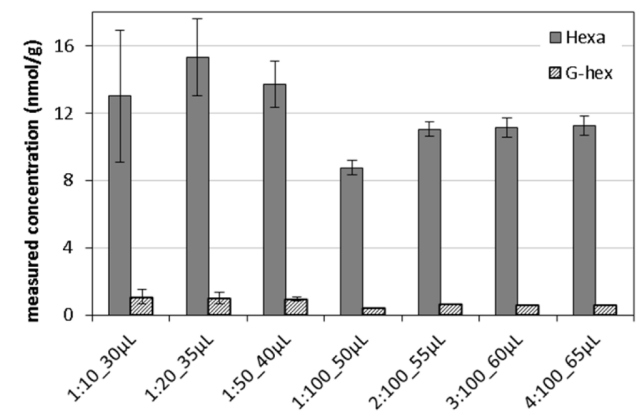

(C)

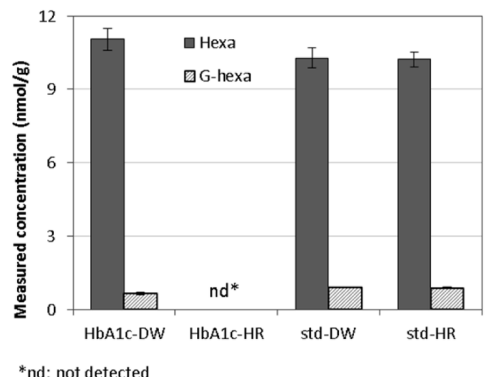

(B)

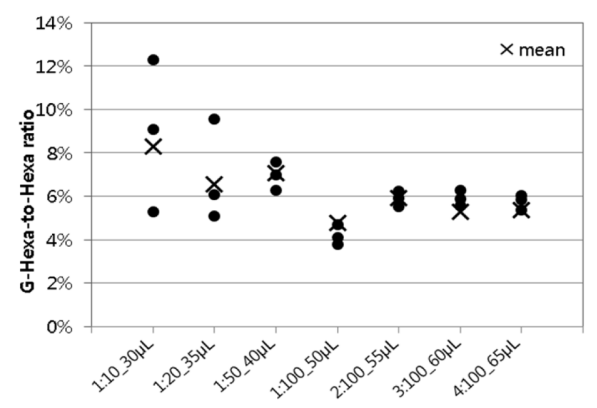

(E)

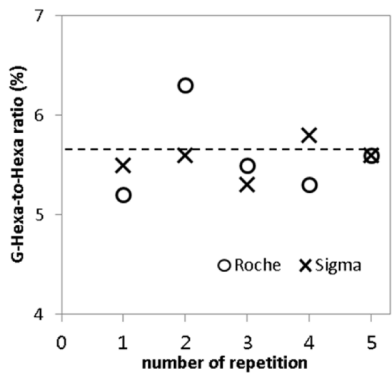

Figure 2. Optimization of enzyme digestion conditions. Measured concentration of Hexa and G-Hexa (A), and G-Hexa-to-Hexa ratio (B) with respect to the Glu-C-to-HbA1c ratio and total sample volume. The Glu-C-to-HbA1c ratios were represented mass ratio of Glu$\mathrm{C}$ and $\mathrm{HbAlc}$ as $x: y$ followed by the total sample volume in microliter. (C), Measured concentration of Hexa and G-Hexa with distilled water (DW) and hemolyzing reagent (HR) as diluents of intact HbAlc. (D), G-Hexa-to-Hexa ratio of five repetitive experiments using Glu-C from different manufacturer, Roche and Sigma. Dashed line represents mean value of results. (E), G-Hexa-to-Hexa ratio when using only Glu-C vs. Glu-C with trypsin.

and patient samples in the clinical laboratory. ${ }^{18}$ That was the reason why they used different dilution reagent: distilled water for the standard but hemolysing reagent for the patient sample. Two reagents were tested as diluents of intact HbA1c. Except dilution solvents, all other experimental conditions were same. Sample preparation procedure was explained in the Experimental section. When using hemolysing reagent, enzyme activity was significantly diminished (Figure 2C). Compared to the standard solution, hemolyzing reagent did not affect LC separation or the MS signal. This will be a point to check when the commutability of HbAlc CRM to routine analytical methods using typical hemolyzing reagents was tested.

There are several commercial Glu-Cs; Glu-C products from Roche (Product No. 114203999001) and Sigma (Product No. P6181) were cross checked (Figure 2D). While there was no significant difference in mean measured values between them in five repetitive experiments, (as depicted by the dashed line in Figure 2D), the reproducibility was better when using Glu-C from Sigma.

Finally, some enzymes become more active when in the presence of other enzymes. The digestion efficacy of hexapeptides in Glu-C alone and in Glu-C combined with trypsin was tested. The added volume and concentration of enzymes were $10 \mu \mathrm{L}$ of $0.2 \mathrm{mg} / \mathrm{mL}$ Glu-C, and $10 \mu \mathrm{L}$ of

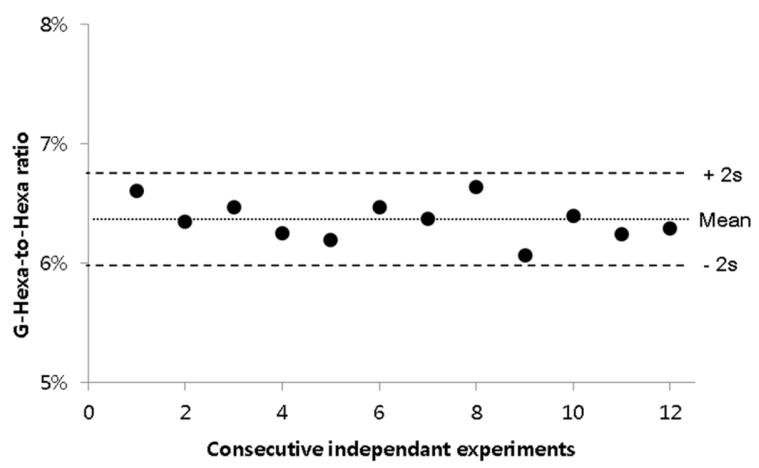

Figure 3. G-Hexa to Hexa ratios with 12 consecutive and independent experiments under the optimized sample preparation procedure in Experimental section.

$0.2 \mathrm{mg} / \mathrm{mL}$ trypsin, respectively. The final sample volume was $50 \mu \mathrm{L}$ of Glu-C only and $60 \mu \mathrm{L}$ of Glu-C and trypsin combined sample. As shown in Figure 2E, no improvement was found when using the combined enzymes.

Under these optimized conditions, repeatability and reproducibility tests were perfomed using twelve repetitions. The optimized sample preparation procedure was explained in the Experimental section. As shown in Figure 3, the relative standard deviation of G-Hexa to Hexa 
ratio was successfully maintained at $2.6 \%$. In summary, throughout the optimization process, some issues in practical experiments, such as total volume, enzyme-tosample mass ratio, enzyme manufacturer, etc., were proposed. As a result, the final measured value was not significantly improved, but reproducibility was improved with optimization (Figures 2A and 2B). Further study for commutability or analytical bias using specific hemolyzing reagents is required (Figure 2C). Additionally, the manufacturer of Glu-C and the use of multiple enzymes with Glu-C did not significantly affect HbAlc quantification results (Figure 2D and 2E).

\section{Conclusions}

Sample preparation procedures were optimized to achieve reliable ID-LC-MS/MS results as a higher order measurement procedure for quantification of $\mathrm{HbAlc}$. Synthetic peptides used for reference materials were also certified with ID-LC-MS/MS to establish traceability to SI units, and some specific parameters were investigated to assure reliability of enzyme digestion. Through this study, analytical throughput was improved by shortening run time, and experimental stability and SI traceability was also achieved. This protocol is suitable for the accurate quantification of $\mathrm{HbA1c}$, and should satisfactorily serve as a reference measurement procedure for certification of CRM and verification of secondary reference materials.

\section{Acknowledgements}

This work was supported by the Korea Research Institute of Standards and Science under the project "Development of Protein Measurement Standards", Grant 14011043.

\section{References}

1. Sacks, D. B. Diabetes Care 2011, 34, 518.

2. Bi, J.; Wu, L.; Yang, B.; Yang, Y.; Wang, J. Anal. Bioanal. Chem. 2012, 403, 549.

3. Bry, L.; Chen, P. C.; Sacks, D. B. Clin. Chem. 2001, 47, 153.

4. Thevarajah, M.; Nadzimah, M. N.; Chew, Y. Y. Clin. Biochem. 2009, 42, 430 .

5. Kobold, U.; Jeppsson, J. O.; Dulffer, T.; Finke, A.; Hoelzel, W.; Miedema, K. Clin. Chem. 1997, 43, 1944.

6. Little, R. R.; Rohlfing, C. L.; Wiedmeyer, H. M.; Myers, G. L.; Sacks, D. B.; Goldstein, D. E. Clin. Chem. 2001, 47, 1985.

7. Goodall, I. Clin. Biochem. Rev. 2005, 26, 5.

8. Little, R. R.; Rohlfing, C. L.; Sacks, D. B. Clin. Chem. 2011, 57, 205.

9. Jeppsson, J. O.; Kobold, U.; Barr, J.; Finke, A.; Hoelzel, W.; Hoshino, T.; Miedema, K.; Mosca, A.; Mauri, P.; Paroni, R.; Thienpont, L.; Umemoto, M.; Weykamp, C. Clin. Chem. Lab. Med. 2002, 40, 78.

10. Finke, A.; Kobold, U.; Hoelzel, W.; Weykamp, C.; Miedema, K.; Jeppsson, J. O. Clin. Chem. Lab. Med. 1998, 36, 299.

11. Kaiser, P.; Akerboom, T.; Ohlendorf, R.; Reinauer, H. Clin. Chem. 2010, 56, 750.

12. Vesper, H. W.; Mi, L.; Enada, A.; Myers, G. L. Rapid Commun. Mass Spectrom. 2005, 19, 2865.

13. Hirokawa, K.; Shimoji, K.; Kajiyama, N. Biotechnol. Lett. 2005, 27, 963.

14. Jeppsson, J. O.; Jerntorp, P.; Sundkvist, G.; Englund, H.; Nylund, V. Clin. Chem. 1986, 32, 1867.

15. Jeong, J. S.; Lim, H. M.; Kim, S. K.; Ku, H. K.; Oh, K. H.; Park, S. R. J. Chromatogr. A 2011, 1218, 6596.

16. Weiss, M.; Manneberg, M.; Juranville, J. F.; Lahm, H. W.; Fountoulakis, M. J. Chromatogr. A 1998, 795, 263.

17. Drapeau, G. R. Methods Enzymol. 1976, 45, 469.

18. van den Ouweland, J. M.; de Keijzer, M. H.; van Daal, H. Clin. Biochem. 2010, 43, 623. 